\title{
Clusters in the inner spiral arms of M 51: The cluster IMF and the formation history ${ }^{\star} \star \star \star$
}

\author{
A. Bik ${ }^{1,2}$, H. J. G. L. M. Lamers ${ }^{1,3}$, N. Bastian ${ }^{1}$, N. Panagia ${ }^{4,5}$, and M. Romaniello ${ }^{6}$ \\ 1 Astronomical Institute, Utrecht University, Princetonplein 5, 3584 CC Utrecht, The Netherlands \\ 2 Astronomical Institute Anton Pannekoek, University of Amsterdam, Kruislaan 403, 1098 SJ Amsterdam, The Netherlands \\ e-mail: bik@astro.uva.nl \\ 3 SRON Laboratory for Space Research, Sorbonnelaan 2, 3584 CA Utrecht, The Netherlands \\ ${ }^{4}$ Space Telescope Science Institute, 3700 San Martin Drive, Baltimore, MD21218, USA \\ e-mail: panagia@stsci.edu \\ 5 On assignment from the Space Science Department of ESA \\ ${ }^{6}$ European Southern Observatory, Karl-Schwarzschild Strasse 2, Garching-bei-Muenchen 85748, Germany \\ e-mail: mromanie@eso.org
}

Received 19 June 2002 / Accepted 16 September 2002

\begin{abstract}
We present the results of an analysis of the HST-WFPC2 observations of the interacting galaxy M 51. From the observations in 5 broadband filters $(U B V R I)$ and two narrowband filters ( $\mathrm{H} \alpha$ and $[\mathrm{OIII}])$ we study the cluster population in a region of $3.2 \times 3.2 \mathrm{kpc}^{2}$ in the inner spiral arms of M 51, at a distance of about 1 to $3 \mathrm{kpc}$ from the nucleus. We found 877 cluster candidates and we derived their ages, initial masses and extinctions by means of a comparison between the observed spectral energy distribution and the predictions from cluster synthesis models for instantaneous star formation and solar metallicity. The lack of [OIII] emission in even the youngest clusters with strong $\mathrm{H} \alpha$ emission, indicates the absence of the most massive stars and suggests a mass upper limit of about 25 to $30 M_{\odot}$. The mass versus age distribution of the clusters shows a drastic decrease in the number of clusters with age, much more severe than can be expected on the basis of evolutionary fading of the clusters. This indicates that cluster dispersion is occurring on a timescale of $10 \mathrm{Myr}$ or longer. The cluster initial mass function has been derived from clusters younger than $10 \mathrm{Myr}$ by a linear regression fit of the cumulative mass distribution. This results in an exponent $\alpha=-\mathrm{d} \log N(M) / \mathrm{d} \log (M)=2.1 \pm 0.3$ in the range of $2.5 \times 10^{3}<M<5 \times 10^{4} M_{\odot}$ but with an overabundance of clusters with $M>2 \times 10^{4} M_{\odot}$. In the restricted range of $2.5 \times 10^{3}<M<2 \times 10^{4} M_{\odot}$ we find $\alpha=2.0 \pm 0.05$. This exponent is very similar to the value derived for clusters in the interacting Antennae galaxies, and to the exponent of the mass distribution of the giant molecular clouds in our Galaxy. To study the possible effects of the interaction of M 51 with its companion NGC 5195 about $400 \mathrm{Myr}$ ago, which triggered a huge starburst in the nucleus, we determined the cluster formation rate as a function of time for clusters with an initial mass larger than $10^{4} M_{\odot}$. There is no evidence for a peak in the cluster formation rate at around 200 to $400 \mathrm{Myr}$ ago within $2 \sigma$ accuracy, i.e. within a factor two. The formation rate of the detected clusters decreases strongly with age by about a factor $10^{2}$ between $10 \mathrm{Myr}$ and $1 \mathrm{Gyr}$. For clusters older than about $150 \mathrm{Myr}$ this is due to the evolutionary fading of the clusters below the detection limit. For clusters younger than $100 \mathrm{Myr}$ this is due to the dispersion of the clusters, unless one assumes that the cluster formation rate has been steadily increasing with time from 1 Gyr ago to the present time.
\end{abstract}

Key words. galaxies: individual: M 51 - galaxies: interactions - galaxies: spiral - galaxies: starburst - galaxies: star clusters

\section{Introduction}

The interaction of galaxies triggers star formation as clearly seen in the young starbursts in interacting galaxies (for reviews

Send offprint requests to: H. J. G. L. M. Lamers,

e-mail: lamers@astro.uu.nl

* Based on observations with the NASA/ESA Hubble Space Telescope, obtained at the Space Telescope Science Institute, which is operated by AURA, Inc., under NASA contract NAS 5-26555.

$\star \star$ The coordinates and the photometry of the objects are available in electronic form at http://astro.uu.nl/ bastian/M51-bik/ and at http://www.edpsciences.org see Kennicutt 1998; Schweizer 1998). One of the best examples is the Antennae system where the interaction is presently going on and star formation and cluster formation occurs on a very large scale in the region between the two merging nuclei (Whitmore et al. 1999).

The way the triggered star formation progresses through an interacting galaxy is best studied from a slightly older interacting system, where the close passage of the companion is over. In such a system one can hope to derive the age distribution of clusters as a function of location in the galaxy and thus measure the time sequence of the induced star and cluster formation as a function of location in the galaxy. 
One of the best systems for this purpose is the interacting sytem of the Whirlpool galaxy (M 51) at a distance of $8.4 \pm 0.6 \mathrm{Mpc}$ (Feldmeier et al. 1997). The M 51 system consists of a grand design spiral galaxy (NGC 5194) interacting with its dwarf companion (NGC 5195). The almost face-on orientation of M 51 allows the observation of its structure in great detail with minimum obscuration by interstellar dust. The interaction of these two galaxies has been modelled by various authors, starting from the fundamental paper by Toomre $\&$ Toomre (1972). These authors derived an age of the closest passage to be $2 \times 10^{8}$ years ago. Later refined models, e.g. by Hernquist (1990), and reviewed by Barnes (1998), have improved this time estimate. The closest approach is now believed to have occurred about 250-400 Myrs ago. The best model is found for a distance of the pericenter of 17 to $20 \mathrm{kpc}$, for a mass ratio of $M / m \approx 2$ and a relative orbit which crosses the plane of M 51 under an angle of about 15 degrees. (For a discussion of the problems with this model and possible improvements, see Barnes 1998). Recently Salo \& Laurikainen (2000) suggested that on the basis of $N$-body simulations that the M 51 system had multiple passages, with the two last at 50-100 Myrs and 400-500 Myrs ago.

Because of its relatively small distance from us, and the fact that we see the M 51 system face one, the system is ideally suited for the study of the progression of cluster formation due to galaxy - galaxy interaction. For this reason we started a series of studies of different aspects of the M 51 system based on HST-WFPC 2 observations in six broad band and two narrow band filters.

The nucleus of M 51 was studied by Scuderi et al. (2002). They found that the core contains a starburst with an age of $410 \pm 140$ Myrs and a total stellar mass of about $2 \times 10^{7} M_{\odot}$ within the central $17 \mathrm{pc}$. This age agrees with the estimated time of closest passage of the companion, so the starburst in the core is most likely due to the interaction with the passing companion. M 51 contains an unresolved nucleus with a diameter smaller than $2 \mathrm{pc}$ and a luminosity of $2 \times 10^{6} L_{\odot}$ (Scuderi et al. 2002).

The bulge, i.e. the reddish region with a size of $11 \times$ $16 \operatorname{arcsec}^{2}=460 \times 680 \mathrm{pc}^{2}$, between the nucleus and the inner spiral arms, was studied by Scuderi et al. (2002) and by Lamers et al. (2002). The bulge is dominated by an old stellar population with an age in excess of 5 Gyrs. The HST-WFPC2 images of the bulge show the presence of dust lanes. The total amount of dust in the bulge is about $2.3 \times 10^{3} M_{\odot}$ and the dust has about the same extinction law and approximately the same gas to dust ratio as our Galaxy (Lamers et al. 2002). This suggests a metallicity close to that in the solar neighbourhood. The HST-WFPC2 images of the bulge of M51 show clearly that the dust is concentrated in structures in the forms of spiral-like dust lanes and a bar that reaches all the way down into the core. Most intriguingly is the discovery of about 30 bright and mainly blue point-like sources in the bulge that are aligned more or less along the spiral-like dust lanes. Lamers et al. (2002) have shown that these are most likely very young massive stars $20<M_{*}<150 M_{\odot}$ with little evidence of associated clusters. This mode of star formation is the result of the peculiar conditions, in particular the destruction of $\mathrm{CO}$ molecules, of the interstellar clouds in the bulge of M 51.

In this study we focus on the clusters at a distance of about 1 to $3 \mathrm{kpc}$ from the nucleus, i.e. near the inner spiral arms. The purpose of the paper is two-fold:

- (a) to determine the cluster intitial mass function, and

- (b) to determine the presence or absence of a starburst period that can be linked to triggering by the passage of the companion. This is not an easy task, because our data will show that the age distribution of the clusters is strongly affected by the disruption of clusters older than about 40 Myrs.

We study the clusters and their properties by identifying point-like sources in the HST-WFPC2 images and measuring their $U B V R I$ magnitudes to obtain their energy distributions. The magnitudes indicate that the sources are clusters instead of single stars. Their energy distributions are compared to cluster evolutionary synthesis models to determine the age, mass and $E(B-V)$ of these clusters.

In Sect. 2 we describe the observations and the data reduction. In Sect. 3 the selection of cluster candidates is discussed. In Sect. 4 we describe the cluster evolutionary synthesis models and in Sect. 5 the fitting procedure for the derivation of the cluster parameters is explained. The mass versus age distribution of the clusters is derived in Sect. 6. In Sect. 7 the initial mass function of the clusters is derived from the sample of clusters younger than $10 \mathrm{Myrs}$. The cluster formation history is studied in Sect. 8. The summary and conclusions are given in Sect. 9.

\section{Observations and reduction}

M51 was observed with $H S T-W F P C 2$ as part of the $H S T$ Supernova INtensive Study (SINS) program (Millard et al. 1999). For this study we use the images taken in the broad band filters $F 336 W(U), F 439 W(B), F 555 W(V), F 675 W(R)$ and $F 814 W(I)$ from the SINS program and in the narrow band filters $F 502 N([\mathrm{OIII}])$ and $F 656 N(\mathrm{H} \alpha)$ from the GO-program of H. C. Ford. The image in $U$ was taken on 1994 May 12, the BVRI images were taken on Jan. 151995 and the [OIII] and $\mathrm{H} \alpha$ images on Jan. 25 1995. The $U$ and $B$ images were split into three and two exposures of $400 \mathrm{~s}$ and $700 \mathrm{~s}$ respectively. The [OIII] and $\mathrm{H} \alpha$ images are split into two exposures of $1200 \mathrm{~s}$ and $500 \mathrm{~s}$ ([OIII]), and $1400 \mathrm{~s}$ and $400 \mathrm{~s}(\mathrm{H} \alpha)$. In the remaining bands one single exposure of $600 \mathrm{~s}$ was taken. The data was processed through the PODPS (Post Observing Data Processing System) for bias removal, flat fielding and dark frame correction.

To remove the cosmic rays from the $U, B[\mathrm{OIII}]$ and $\mathrm{H} \alpha \mathrm{im}$ ages, we used the STSDAS task crrej for combining the available exposures. For the VRI images, where only one exposure is available, we used a procedure called "Cosmic Eraser". This procedure combines the IRAF tasks cosmicrays and imedit to reject as carefully as possible the cosmic rays. The automatic detection of cosmic rays with the task cosmicrays is based upon two parameters, a detection threshold and a flux ratio. The first parameter enables the detection of all the pixels with a value larger than the average value of the surrounding pixels. The flux ratio is defined as the percentage of the average value of the 
four neighboring pixels (excluding the second brightest pixel) to the flux of the brightest pixel. This parameter allows a classification of the detected objects: cosmic ray or star. Training objects are used to determine the flux ratio carefully. These training objects are labeled by the user to be a cosmic ray or a star. With imedit the detected cosmic rays signals are replaced by an interpolation of a third order surface fit to the surrounding pixels.

After the correction for the cosmic rays, the images were corrected for bad pixels using the hot pixel list from the STScI WFPC2 website in combination with the task warmpix. Corrections for non-optimal charge transfer efficiency on the CCD's of the WFPC2 camera were applied using the formulae by Whitmore \& Heyer (1997).

With the task daofind from the DAOPHOT package Stetson (1987), we identified the point sources on the image. We performed aperture photometry on these sources, also with the DAOPHOT package. We used an aperture radius of 3 pixels. The sky background was calculated in an annulus with internal and external radius of 10 and 14 pixels respectively. We only selected the point sources with an uncertainty smaller than 0.2 in the magnitude. Photometric zeropoints were obtained from table 28.2 of the HST Data Handbook (Voit 1997), using the VEGAMAG photometric system (Holtzman et al. 1995).

The aperture correction was measured for a number of isolated, high $S / N$ point sources on each WFPC2-chip. This output was adopted for all the other point sources on the chip. Following Holtzman et al. (1995) we have normalized the aperture correction to $1^{\prime \prime}$ (10 WF pixels). The aperture corrections we found are between -0.24 and $-0.37 \mathrm{mag}$. This is larger than the aperture corrections for stars $\approx-0.17$ mag (Holtzman et al. 1995), which means that the detected point sources are fairly well resolved.

We adopt a distance of $d=8.4 \pm 0.6 \mathrm{Mpc}$ (Feldmeier et al. 1997), which corresponds to a distance modulus of 29.62. At this distance, $1^{\prime \prime}$ corresponds to a linear distance of $40.7 \mathrm{pc}$, which means that an HST-WFC pixel of $0.1^{\prime \prime}$ corresponds to $4.1 \mathrm{pc}$.

\section{Selection of the clusters}

The $U$-band images were taken in 1994, 8 months earlier than the images in the other passbands. The orientation of HSTWFPC2 was not the same at the two epochs. In the 1995 $B V R I$-images the nucleus of M 51 was in the center of the PCimage. The $U$-image was centered on SN1994I (that was 15" off from the nucleus) so that the nucleus was near the edge of the PC-image. The orientation of the WFPC2-images is shown in Fig. 1.

For our analysis only the part of $W F 4$-chip of the $U$ image that overlap with the WF2-chip of the BVRI images is used (see Fig. 1). To identify the various sources, we obtained a position of the sources in $U$ and transformed these positions to the coordinate frame of the $B V R I$ images. The orientation of the [OIII] and $\mathrm{H} \alpha$ images is very similar to those of the $B V R I$ images. We transformed these narrow band images to the same orientation as the $B V R I$ images.
We applied our analysis of the cluster energy distributions to the sources that were detected in the images of at least three of the $B V R I$ broad-band filters with a magnitude uncertainty smaller than 0.2 in each band. To this purpose we compared the positions of all detected sources in the BVRI images. If objects occur in at least three images within a position tolerance of 2 pixels they are selected for our study. This tolerance was chosen on the basis of tests with clearly identifiable sources that were observed in most bands. For the sources detected in at least three images we then checked for the presence of a source in the $U$ image, in the region where the $U$ image and the $B V R I$ images do overlap (the dark grey region in Fig. 1). If a source was not detected in a particular band, we adopted a conservative lower limit for the magnitude in that band (see below). This was not always possible for the $U$-magnitude, because many of the sources detected in the BVRI bands are located in an area that was not covered by the $U$-image. As a consequence, we have no information on the $U$-magnitude for many of the sources.

The location of the sources in the WF2-chip is shown in Fig. 2. The overlay of the sources on the $V$ band image clearly shows that the clusters are concentrated in or near the spiral arms at a distance of about $1.5 \mathrm{kpc}$ from the nucleus of M 51 . The number of sources, detected in the various bands, is listed in Table 1 . The sample contains a total of 877 sources for which we have reliable photometry $(\sigma<0.20 \mathrm{mag})$ in at least three bands.

Figure 3 shows the magnitude distribution of the sources in the various bands. All distributions show a slow increase in numbers towards fainter objects, a maximum and a steep decrease towards even fainter sources. The slow increase to fainter sources reflects their luminosity function. The steep decrease to high magnitudes is due to the detection limit. This detection limit is not a single value for each band, because of the variable background in the $W F 2$ field due to the spiral arms. The maximum of the distributions are near $U \simeq 20.0, B \simeq 22.0$, $V \simeq 22.0, R \simeq 21.5$ and $I \simeq 21.0$. These values will be adopted as conservative lower limits for the magnitudes of sources that were not detected in any given band.

All the objects fall in the visual magnitude range of $16.5<$ $V<23.6$. For distance modulus of 29.62 this corresponds to an absolute magnitude range of $-12.1<M_{V}<-6.0$ in the case of zero extinction and $-12.6<M_{V}<-6.5$ in case of moderate extinction with $E(B-V) \simeq 0.17$. This means that the faintest objects could be either very bright stars or small clusters. The vast majority of the selected point sources are so bright that they must be clusters. (See Sect. 5.6.)

\subsection{The $\mathrm{H} \alpha$ and [OIII] magnitudes}

The magnitudes in the narrow-band images of filters $F 656 N$ $(m(\mathrm{H} \alpha)$ ) and $F 502 N(m[\mathrm{OIII}])$ can be compared with those of the wide-band filters $F 675 W(R)$ and $F 555 W(V)$ respectively to derive a measure of the equivalent width of the $\mathrm{H} \alpha$ and [OIII] lines. When the magnitudes are expressed in the HST-system, the magnitude differences $R-m(\mathrm{H} \alpha)$ and $V-m$ [OIII] are by definition equal to 0.0 if the spectrum of a source is a featureless, 

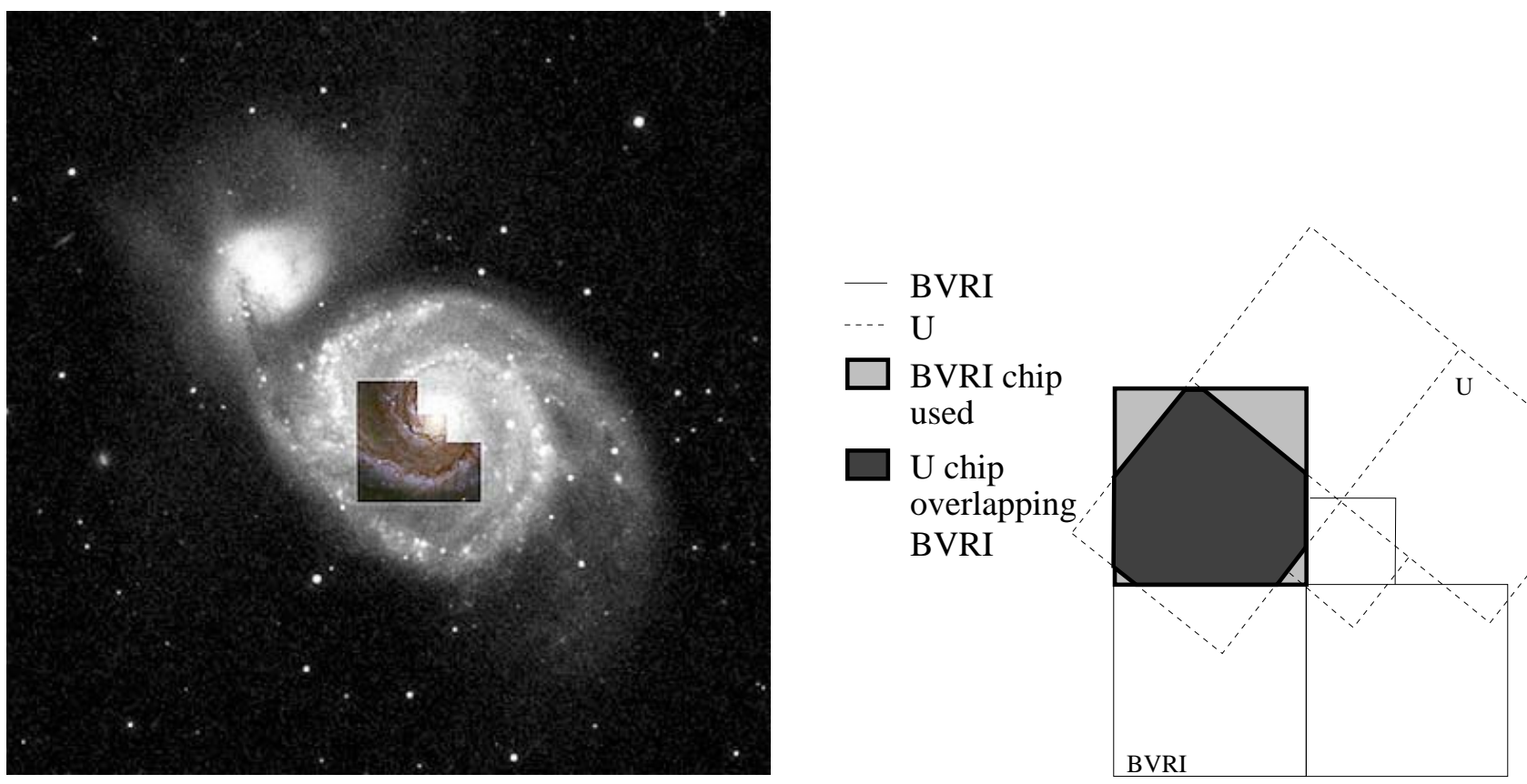

Fig. 1. The location of the $W F P C 2$ camera when the $B V R I$ images were taken, superimposed on an optical image of M 51. Right: the orientation of the WFPC2 camera when the $B V R I$ and the $U$ images were taken. The grey area indicates the location of the $W F 2$ chip, covering an area of $3.25 \times 3.25 \mathrm{kpc}$ (for an adopted distance of $8.4 \mathrm{Mpc}$ ), that was used in this analysis. Its exact location and orientation are given in Fig. 2 . The dark grey region shows the overlap of the $B V R I$ and $U$ images.

Table 1. Numbers of point sources detected in the different $W F P C 2-$ HST-images.

\begin{tabular}{cccccr}
\hline \hline $\begin{array}{c}F 336 W \\
(U)\end{array}$ & $\begin{array}{c}F 439 W \\
(B)\end{array}$ & $\begin{array}{c}F 555 W \\
(V)\end{array}$ & $\begin{array}{c}F 675 W \\
(R)\end{array}$ & $\begin{array}{r}F 814 W \\
(I)\end{array}$ & $\begin{array}{r}\text { number } \\
\text { objects }\end{array}$ \\
\hline $\mathrm{d}$ & $\mathrm{d}$ & $\mathrm{d}$ & $\mathrm{d}$ & $\mathrm{d}$ & 76 \\
1 & $\mathrm{~d}$ & $\mathrm{~d}$ & $\mathrm{~d}$ & $\mathrm{~d}$ & 366 \\
$\mathrm{n}$ & $\mathrm{d}$ & $\mathrm{d}$ & $\mathrm{d}$ & $\mathrm{d}$ & 109 \\
$\mathrm{~d}$ & $\mathrm{l}$ & $\mathrm{d}$ & $\mathrm{d}$ & $\mathrm{d}$ & 30 \\
1 & 1 & $\mathrm{~d}$ & $\mathrm{~d}$ & $\mathrm{~d}$ & 144 \\
$\mathrm{n}$ & $\mathrm{l}$ & $\mathrm{d}$ & $\mathrm{d}$ & $\mathrm{d}$ & 39 \\
$\mathrm{~d}$ & $\mathrm{~d}$ & $\mathrm{~d}$ & $\mathrm{~d}$ & 1 & 21 \\
$\mathrm{l}$ & $\mathrm{d}$ & $\mathrm{d}$ & $\mathrm{d}$ & 1 & 78 \\
$\mathrm{n}$ & $\mathrm{d}$ & $\mathrm{d}$ & $\mathrm{d}$ & 1 & 14 \\
\hline & & & & & 877 \\
\hline
\end{tabular}

d means "detected", 1 means "lower magnitude limit" (i.e. the object is fainter than the detection limit) and n means "not observed".

flat continuum (i.e. the spectrum of the source is a continuum $F_{\lambda}=$ const. without a photospheric $\mathrm{H} \alpha$ absorption, and there is no HII region around the star). The differences $R-m(\mathrm{H} \alpha)$ and $V-m[\mathrm{OIII}]$ are either positive or negative if the source spectrum has an emission or an absorption line, respectively, falling in the narrow band filter, independently of the interstellar extinction. With the Vega-system magnitudes one has to fold in both the non-zero spectral slope and the discrete features present in $\alpha$ Lyrae's spectrum. Therefore, a featureless flat continuum would correspond to $R-m(\mathrm{H} \alpha) \simeq+0.08$ and $V-m[\mathrm{OIII}] \simeq-0.26$ colors in the Vega-system.

Figure $4 \mathrm{a}$ shows the $m(\mathrm{H} \alpha)$ versus $R$ and Fig. 4b shows $m$ [OIII] versus $V$ for the subset of sources for which we could

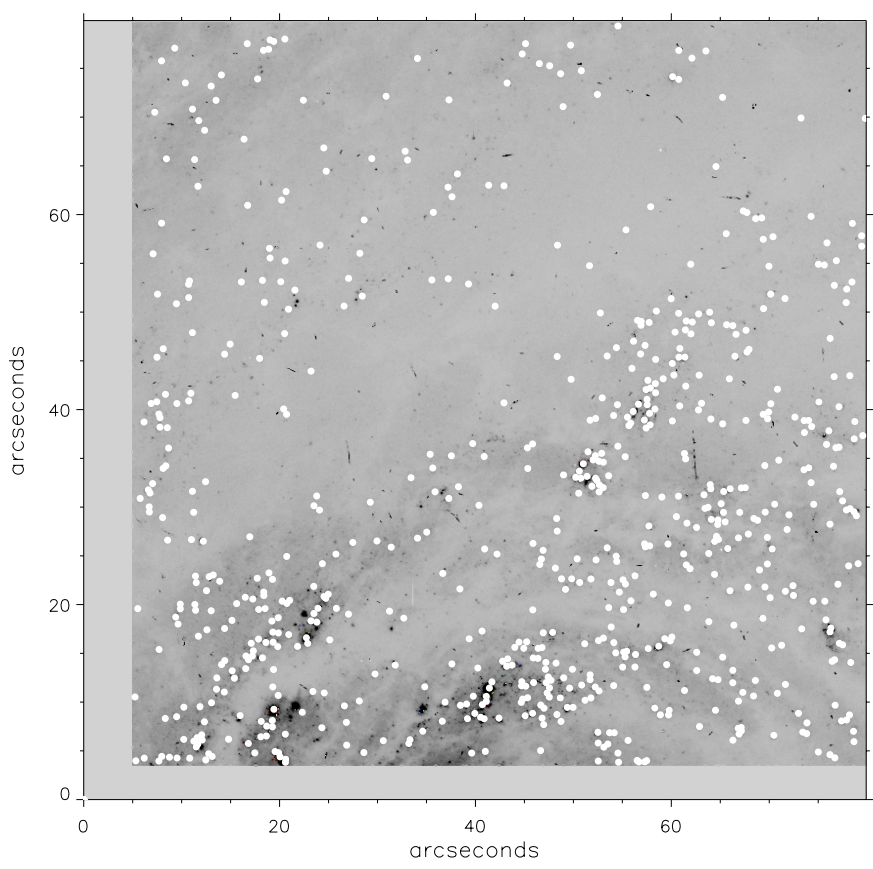

Fig. 2. Left: the location of all the detected point sources in the WF2 chip superimposed on the $V$-band image. This image is rotated 90 degrees clockwise compared to Fig. 1. The coordinates of the lower-left and upper-right corners of the image are: pixel $(1,1): \operatorname{RA}(2000)=13^{\mathrm{h}} 29^{\mathrm{m}} 55^{\mathrm{s}} .90, \operatorname{Dec}(2000)=47^{0} 11^{\prime} 27^{\prime \prime} \cdot 2$. and pixel 800, 800: RA(2000) $=13^{\mathrm{h}} 29^{\mathrm{m}} 57^{\mathrm{s}} .94, \operatorname{Dec}(2000)=47^{0} 13^{\prime} 16^{\prime \prime} 6$.

measure these magnitudes with an accuracy better than 0.2. The figure shows that many sources have $\mathrm{H} \alpha$ emission $(m(\mathrm{H} \alpha)<R)$ but none (!) of the sources has [OIII] emission (note that 

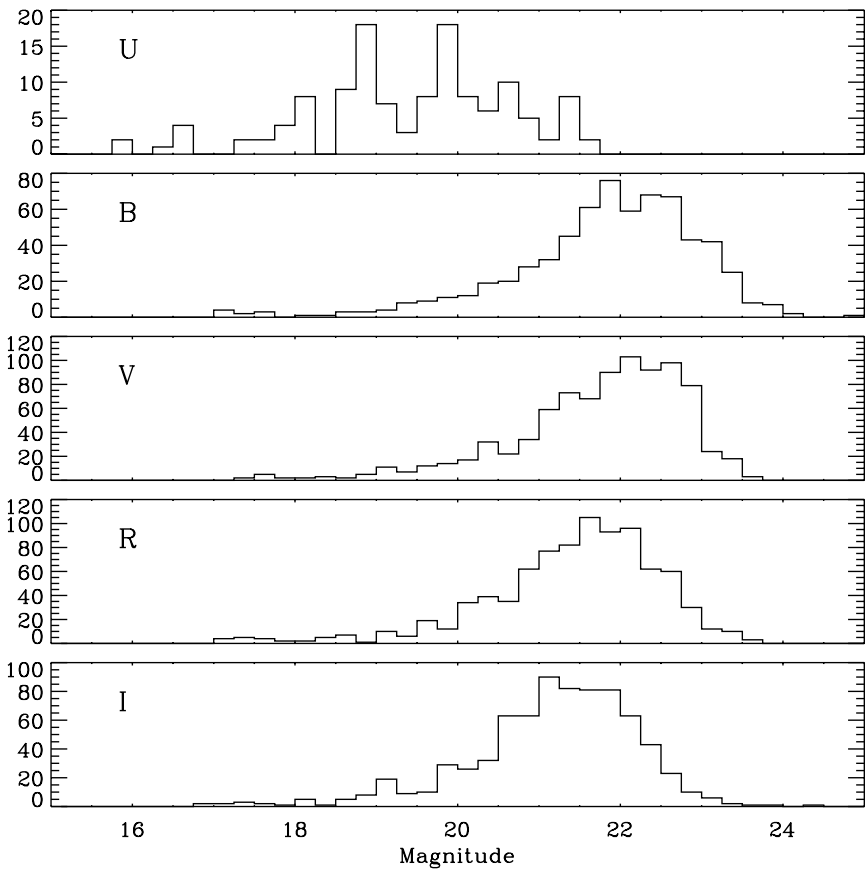

Fig. 3. The histogram of the magnitudes in the different bands. The slow increase at bright magnitudes is due to the luminosity function and the steep decrease at fainter magnitudes is due to the detection limit.

$m[\mathrm{OIII}]$ is slightly fainter than the $V$ magnitude as appropriate for complete absence of an emission line). The lack of [OIII] emission indicates that the far-UV flux, at $\lambda<350 \AA$ i.e. at energies higher than the second ionization potential of oxygen, is quite low. For main sequence stars with $L>2.5 \times 10^{5} L_{\odot}$ (i.e. with $M>30 M_{\odot}$ and $T_{\text {eff }}>33000 \mathrm{~K}$ ), surrounded by an HII region with approximately solar abundances, we would expect $V-m[\mathrm{OIII}]>R-m(\mathrm{H} \alpha)$. We will show later, on the basis of the study of the energy distributions, that many of the objects are very young clusters with ages less than a $10 \mathrm{Myr}$. The lack of [OIII] emission shows that these clusters do not contain stars with $T_{\text {eff }}$ above about $30000 \mathrm{~K}$, which corresponds to about 25 to $30 M_{\odot}$ (e.g. Chiosi \& Maeder 1986). We can exclude the possibility that the lack of [OIII] emission be due to just a high metallicity, which could reduce the electron temperature in an HII region and weaken optical forbidden lines considerably, without requiring a lower effective temperature, or, equivalently, a low upper mass cutoff. This is because in many HII regions in M 51 and in other metal-rich spiral galaxies not only are the [OIII] lines faint or absent but also the HeI lines are unusually faint relative to Balmer lines (Panagia 2000; Lenzuni $\&$ Panagia, in preparation). This result indicates that He is only partially ionized and, therefore, that the ionizing radiation field is indeed produced exclusively by stars with effective temperatures much lower than $33000 \mathrm{~K}$. So we conclude that the upper mass limit for clusters in the inner spiral arms of M 51 is about 25 to $30 M_{\odot}$.
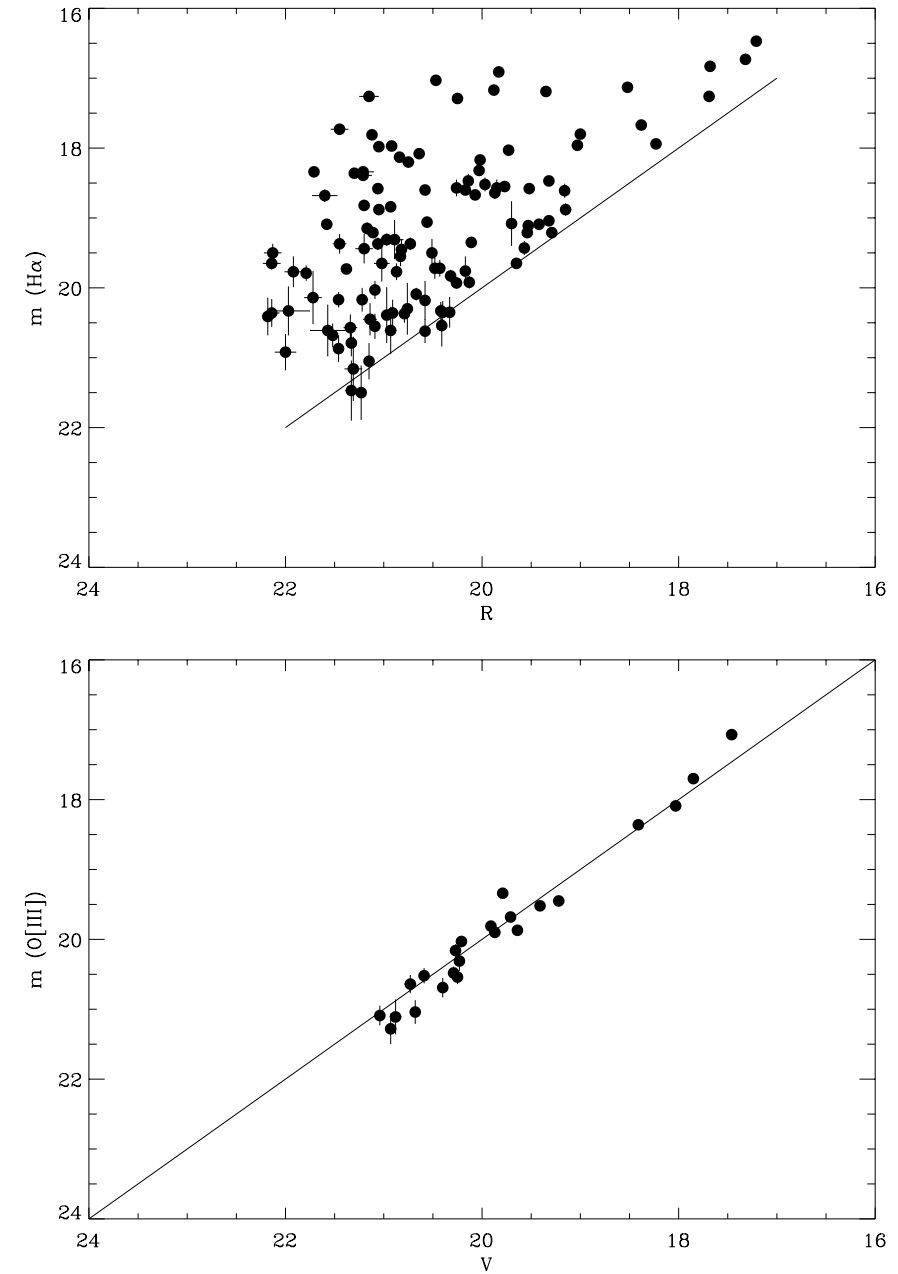

Fig. 4. The top figure shows $m(\mathrm{H} \alpha)$ versus $R$ and the lower figure shows $m[\mathrm{OIII}]$ versus $V$ for objects were these magnitudes could be determined with an accuracy better than $0.2 \mathrm{mag}$. Notice that many objects have $\mathrm{H} \alpha$ emission, but no object has detectable [OIII] emission.

\section{Cluster evolution models}

To determine the age, initial mass and $E(B-V)$ of the clusters, we compare the observed energy distributions with energy distributions derived from theoretical cluster evolutionary synthesis models. We used two sets of models: the Starburst99 models for ages up to $1 \mathrm{Gyr}$, and the Frascati models for ages of 10 Myr to 5 Gyr. In this section the models are described.

\subsection{Starburst99 cluster evolution models}

To determine the age, initial mass and $E(B-V)$ of the clusters, we compare the observed energy distributions with energy distributions derived from theoretical cluster evolutionary synthesis models. We used two sets of models: the Starburst99 models for ages up to $1 \mathrm{Gyr}$, and the Frascati models for ages of 10 Myr to 5 Gyr. In this section the models are described. 


\subsection{Starburst99 cluster evolution models}

We compared the observed spectral energy distributions (SED) of our objects with the cluster evolutionary synthesis models of Leitherer et al. (1999), i.e. the Starburst99 models for instantaneous star formation. In these models, the stellar atmosphere models of Lejeune et al. (1997) are included. For stars with a strong mass loss the Schmutz et al. (1992) extended model atmospheres are used. The stellar evolution models of the Geneva group are included in these models. For our analysis we used the models of the Spectral Energy Distributions (SEDs).

In the cluster models the stars are assumed to be formed with a classical Salpeter IMF-slope of $\mathrm{d} \log (N) / \mathrm{d} \log (M)=$ -2.35 . The lower cut-off mass is $M_{\text {low }}=1 M_{\odot}$. We adopt two values for the upper cut-off mass: the standard value of $M_{\text {up }}=100 M_{\odot}$, and the value of $M_{\text {up }}=30 M_{\odot}$. This last value is adopted because it is suggested by the lack of [OIII] emission (see Sect. 3.1). The total initial mass of all models is $10^{6} \mathrm{M}_{\odot}$. Leitherer et al. (1999) present models for 5 different metallicities, from $Z=0.001$ to $Z=0.040=2 \times Z_{\odot}$. Observations of HII-regions have shown that the metallicity of the inner region of M 51 is approximately $2 Z_{\odot}$ or slightly higher (e.g. Diaz et al. 1991; Hill et al. 1997). Therefore we adopt the models with $Z=0.020$ and 0.040 . The almost solar metallicity agrees with the study of Lamers et al. (2002), who found that the extinction properties and the gas to dust-ratio in the bulge of M 51 is very similar to that in the Milky Way. So we use four sets of models, denoted by the pair $\left(Z, M_{\text {up }}\right)$ of $(0.02,100),(0.02,30)$, $(0.04,100)$ and $(0.04,30)$. For these combinations we calculated the SEDs of the cluster models, taking into account the nebular continuum emission.

To obtain the absolute magnitudes from the theoretical spectral energy distributions in the five broad-band filters and the two narrow-band filters we convolved the predicted spectra of the Starburst99 models with the WFPC2 filter profiles. The spectrum of Vega was also convolved with the filter functions in order to find the zero-points of the magnitudes in the VEGAMAG system. For each combination of $Z$ and $M_{\text {up }}$ we calculated the SED of 195 models in the range of 0.1 Myr to $1 \mathrm{Gyr}$, with time steps increasing from $0.5 \mathrm{Myr}$ for the youngest models to $10 \mathrm{Myr}$ for the oldest models.

The adopted lower mass limit of $1 M_{\odot}$ for the cluster stars has consequences for the derived masses of the clusters. If the IMF with a slope of -2.35 has a lower mass limit of $0.2 M_{\odot}$, as for the Orion Nebula Cluster (Hillenbrand 1997), the derived masses of the clusters would be a factor 2.09 higher for an upper limit of $30 M_{\odot}$. If the lower limit is $0.6 M_{\odot}$, the mass of the clusters will be a factor 1.28 higher than those of the Starburst 99 models for $M_{\text {up }}=30 M_{\odot}$. We will take this effect into account in the determination of the cluster masses.

Some of the theoretical energy distributions in the WFPC2 wide-band filters used in this study are shown in Fig. 5, for clusters with an initial mass of $10^{6} M_{\odot}$ at the distance of M 51 . During the first 5 Myrs the UV flux remains high because most of the O-type stars have main sequence ages longer than $5 \mathrm{Myr}$. Between 5 and 10 Myr the O-type stars disappear. This results in a general decrease at all magnitudes, except in the $I$ band where the red supergiants start to dominate. At later ages the

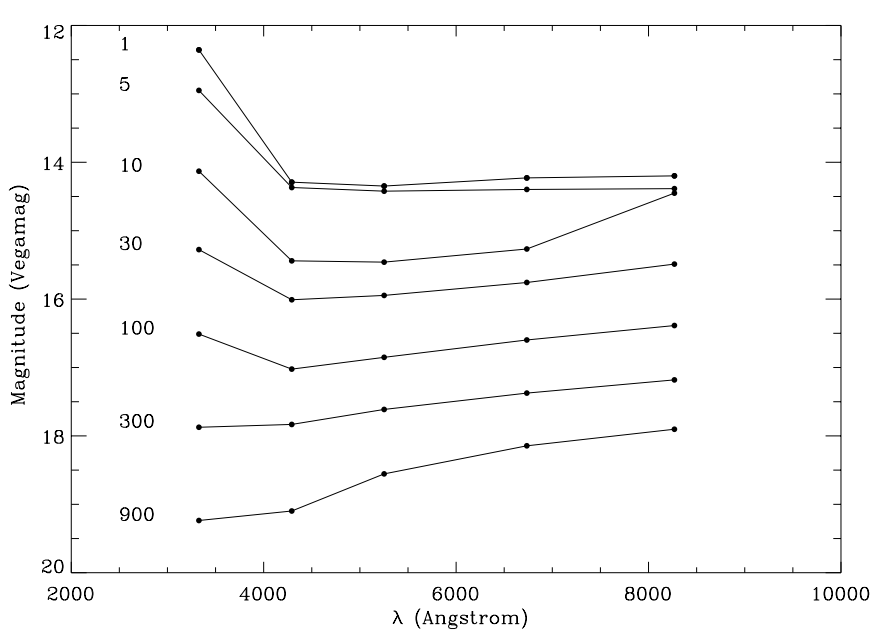

Fig. 5. Examples of some characteristics of the theoretical energy distributions, in magnitude versus wavelength ( $\AA$ ) of the UBVRI filters (from left to right), for STARBURST99 clusters with an initial mass of $10^{6} M_{\odot}$ at the distance of M 51. The age of the cluster in Myrs is indicated. The evolutionary variation is discussed in Sect. 4.1.

flux decreases at all wavelengths as stars end their lives. After 900 Myrs only stars of types late-B and later have survived.

Figure 5 shows that at any wavelength in the observed range the flux decreases with time. This fading of the clusters indicates that the detection limit of the clusters will gradually shift to those of higher mass as the clusters age. This is because the flux of a cluster (for a given stellar initial mass function) at any age and wavelength is proportional to the initial number of stars. The more massive the initial cluster, the brighter the cluster and the longer the flux will remain above the detection limit as the cluster fades due to stellar evolution. For any given detection limit, we can calculate the lower mass limit of the observable cluster as a function of age.

Figure 6 shows the relation between the initial mass of a cluster and its age when it fades below the detection limit, derived from the Starburst 99 models. The $R$-magnitude $(R)$ of a cluster of initial mass $M_{i}$ without extinction at the adopted distance of $8.4 \mathrm{Mpc}$ of M $51(d m=29.62)$ is related to the absolute $R$-magnitude $\left(M_{R}\right)$ predicted for the Starburst 99 models of $10^{6} M_{\odot}$ by

$R(t)=M_{R}(t)+29.62-2.5 \times \log \left(M / 10^{6}\right)$.

So, for a given detection limit $R_{\lim }$ the critical mass $M_{\lim }$ of a cluster that can be detected at age $t$ is given by

$\log M_{\lim }(t)=6+0.4 \times\left(M_{R}(t)+29.62-R_{\lim }\right)$.

We have adopted the $R$ magnitudes to calculate the observed lower limits of the distributions for two reasons: (a) all clusters were detected in the $R$ band and (b) the effect of extinction is small in the $R$ band. The resulting relation between $M_{\text {lim }}$ and age is shown in Fig. 6 for detection limits of $R_{\text {lim }}=22.0$ and $23.0 \mathrm{mag}$. We will use these limits later, in Sect. 5, to explain the distribution of clusters in the mass versus age diagram.

Figure 7 shows the variations of the colours of the Starburst 99 models for solar metallicity and with an upper mass limit of $30 M_{\odot}$. The figure shows that the colours do not change 


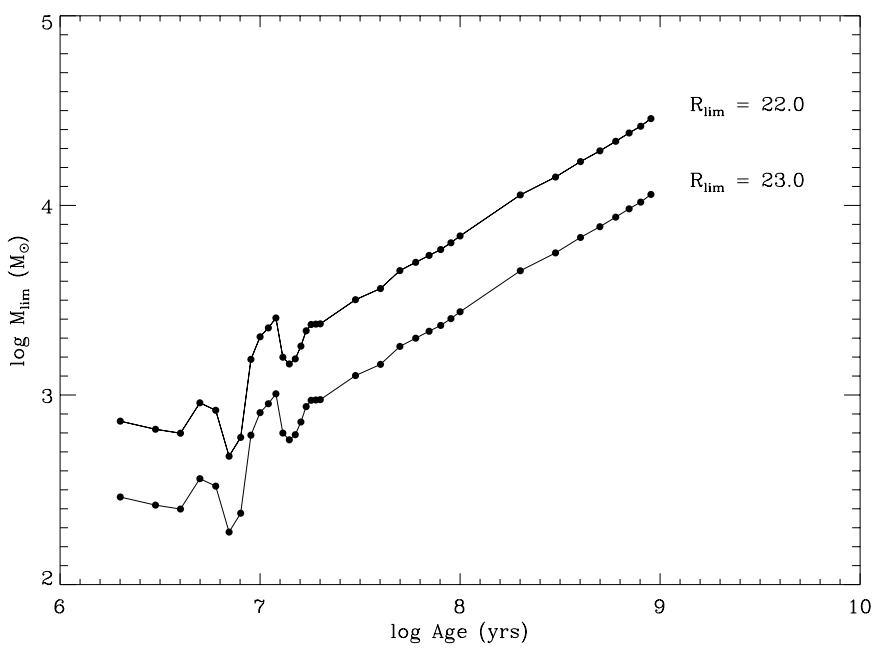

Fig. 6. The relation between the critical initial mass $M_{\text {lim }}$ and age, derived for two values of the $R$-band magnitude limit; 22.0 and 23.0 mag. It is calculated from the Starburst99 cluster models, for a distance of 8.4 Mpc and no extinction. Only clusters with an initial mass higher than $M_{\text {lim }}$ can be detected as faint as $R_{\text {lim }}$ at time $t$. Alternatively, clusters with a mass $M_{\text {lim }}$ can only be detected down to a magnitude $R_{\lim }$ when they are younger than the age $t$, indicated by these relations.

monotonically, but that there are peaks and dips, especially in the age range of $6.5<\log (t)<7.3$. These are related to the phases of the appearance of red supergiants that also produced the dips in the limiting magnitude curves of Fig. 6.

\subsection{The Frascati models}

Since the Starburst99 models only cover the age range up to $1 \mathrm{Gyr}$, we also used synthetic cluster models for older ages from the Frascati group. These "Frascati-models" were calculated by Romaniello (1998) from the evolutionary tracks of Brocato \& Castellani (1993) and Cassisi et al. (1994) using the HST-WFPC2 magnitudes derived from the stellar atmosphere models by Kurucz (1993). These models are for instantaneous formation of a cluster of solar metallicity stars in the mass range of 0.6 to $25 M_{\odot}$, with a total initial mass of $50000 M_{\odot}$, distributed according to Salpeter's IMF. These models cover an age range of 10 to 5000 Myr. There are 48 models with timesteps increasing from $10 \mathrm{Myr}$ for the youngest ones to $500 \mathrm{Myr}$ for the oldest ones. We have increased the magnitudes of the Frascati models by -3.526 mag in order to scale these models to a total mass of $10^{6} M_{\odot}$ in the mass range of 1.0 to $25 M_{\odot}$; i.e. $-3.25 \mathrm{mag}$ correction for the conversion from $5 \times 10^{4}$ to $1 \times 10^{6} M_{\odot}$, and 0.279 mag correction for the conversion from a lower stellar mass limit of $0.6 M_{\odot}$ to $1.0 M_{\odot}$. In this way the masses can be compared directly with those of the Starburst 99 models.

The Starburst 99 models are expected to be more accurate for the younger clusters, because they are based on the evolutionary tracks of the Geneva-group which include massive stars. The Frascati models are expected to be more accurate for the old clusters, because they include stars with masses down to $0.6 M_{\odot}$.

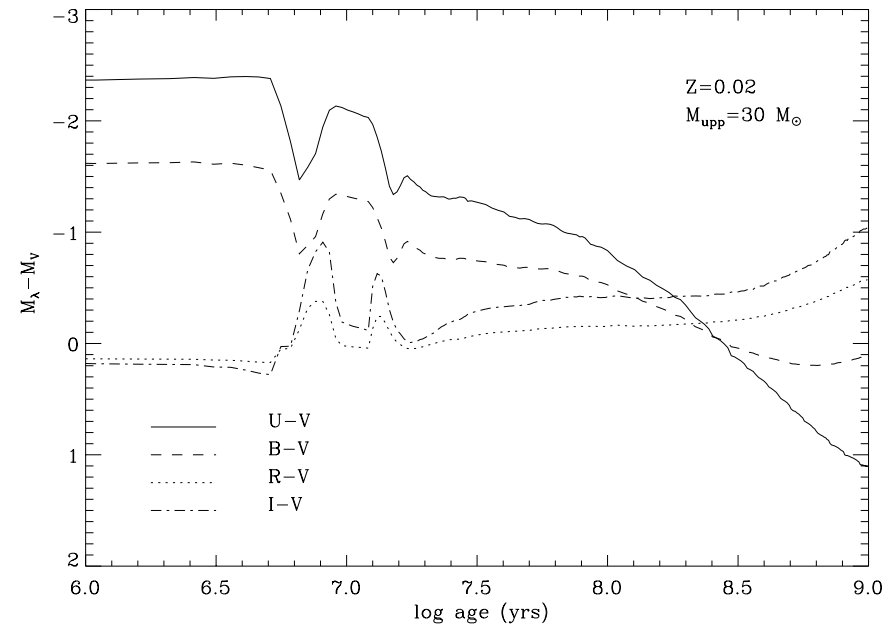

Fig. 7. The variations of colours with age of the Starburst99 models of $Z=0.02$ and an upper mass limit of $30 M_{\odot}$. Notice the periods of rapid variability near the peaks and dips around $t \simeq 6$ and 16 Myrs, $\log (t) \simeq 6.8$ and 7.2.

\subsection{The extinction curve}

To determine the values of $E(B-V)$ of the clusters we assume the mean galactic reddening law. Lamers et al. (2002) and Scuderi et al. (2002) have shown that the extinction law of respectively the bulge and the core of M 51 agree very well with the galactic law. The values of $R_{i}=A_{i} / E(B-V)$ for the HST-WFPC2 filters have been calculated by Romaniello (1998). They are respectively 4.97, 4.13, 3.11, 2.41 and 1.91 for the $U, B, V, R$ and $I$ bands.

In the fitting of the energy distributions we did not take into account the disruption of clusters. We assumed that the stars contribute to the total energy distribution of the clusters until the cluster is completely dissolved and is no longer detectable as a point source. This is a reasonable assumption because we measured the magnitudes in a circle of $12 \mathrm{pc}$ radius (see Sect. 2).

\section{Fitting the observed energy distributions to observed cluster models}

\subsection{The fitting procedure}

We have fitted the energy distributions of the clusters with good photometry in four or more filters, viz. UBVRI, BVRI, UVRI and $U B V R$ (see Table 1), with the energy distributions of the cluster evolutionary synthesis models, discussed above, using a three dimensional maximum likelihood method. The three fitting parameters are: the age of the cluster $(t)$, the reddening $(E(B-V))$ and the initial mass of the cluster $(M)$. For the clusters detected in only three filters we reduce the parameter space and make a two dimensional maximum likelihood fit. For objects not detected in a band, we adopted the magnitude lower limits (described in Sect. 3) to check which fits were acceptable ${ }^{1}$.

${ }^{1}$ We will refer to this method in subsequent papers as the "3(2)DEF- method", i.e. the three (or two) dimensional energy distribution fitting". 


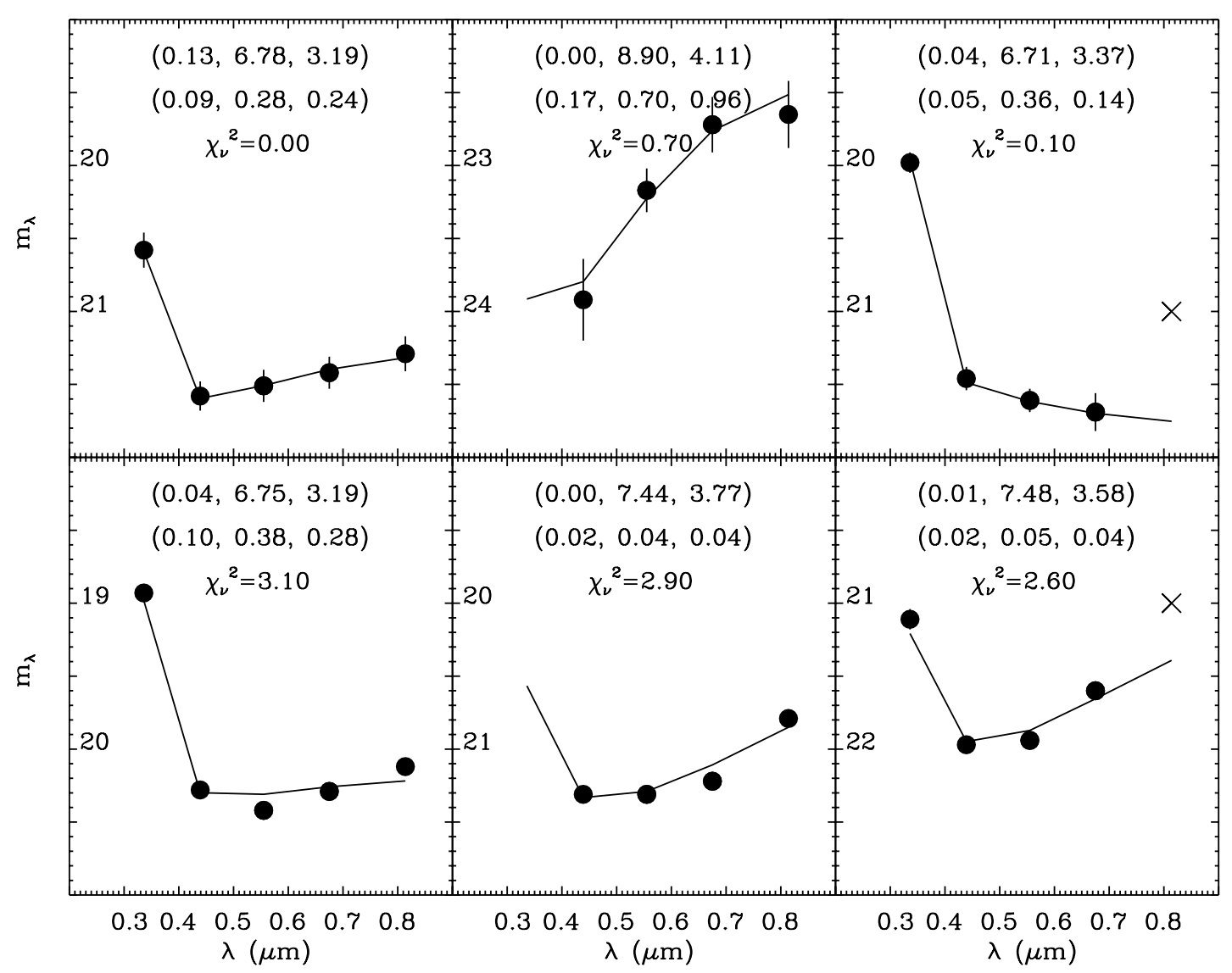

Fig. 8. Example of some fits. The filled dots represent the observed energy distributions of objects measured in at least four bands, with their error bars in magnitude versus wavelength (in $\mu \mathrm{m}$ ). In several cases the error bars are smaller than the size of the dots. Crosses indicate magnitude lower limits. The parameters of the fits are indicated in an array $\left(E(B-V), \log (t), \log \left(M_{\mathrm{cl}}\right)\right)$. The second array gives the uncertainties in these parameters. The line is the best fit obtained with the maximum likelihood method. Top figures: fits with $\chi_{v}^{2}<1$; bottom figures: fits with $\chi_{v}^{2} \simeq 3$. Notice that the uncertainty of the fit parameters depends not only on $\chi_{v}^{2}$ but also strongly on the quality of the data. For instance, the errors in the magnitudes of the last two clusters are very small, so that the fit parameters are accurate, despite the high value of $\chi_{v}^{2}$.

\subsection{Three dimensional maximum likelihood method}

This method was applied to sources that are detected in at least four bands. We fitted the observed energy distributions with those predicted for the Starburst 99 cluster models and the Frascati models (see Sect. 4.2). For each model-age we have two fitting parameters $M$ and $E(B-V)$. The cluster models are for an initial cluster mass of $10^{6} M_{\odot}$. For other masses the flux simply scales $M / 10^{6} M_{\odot}$. We have adopted an uncertainty in the model fluxes of 5 percent $(0.05 \mathrm{mag})$ in all bands.

To reduce the range in masses in the parameter space, we make an initial guess of the cluster mass based on the observed magnitudes, since the distance to M51 is known. By calculating the average difference in magnitude - weighted by the errors - between the theoretical energy distribution for $10^{6} M_{\odot}$ and the observed one, we have a good first approximation of the mass of the cluster:

$M_{\text {guess }}(E(B-V), \tau) \simeq \frac{\sum_{\lambda} 1 / \sigma_{\lambda}\left\{m_{\lambda}^{\text {obs }}-m_{\lambda}^{\bmod }(E(B-V), \tau)\right\}}{\sum_{\lambda} 1 / \sigma_{\lambda}}$

with $E(B-V)$ between 0.0 and 2.0 in steps of 0.02 , where $\sigma$ is the uncertainty in the magnitude. This is a reasonable range, compared to the average colour excess for the bulge of 0.2 found by Lamers et al. (2002). With this initial guess we then make a three dimensional likelihood analysis, using a mass range from $1 / 1.5$ to 1.5 times the initial mass estimate in steps of 0.004 dex. The extinction was varied between 0.0 and 2.0 in steps of 0.01 and the age was varied between $0.1 \mathrm{Myr}$ and 1 Gyr for the Starburst 99 models and between 10 Myr to 5 Gyr for the Frascati models.

For every fit we obtain a value for the reduced $\chi^{2}$, i.e. $\chi_{v}^{2}=$ $\chi^{2} / v$, where $v$ is the number of free parameters i.e. the number of the observed data points minus the number of parameters in the theoretical model. For a good fit, $\chi_{v}^{2}$ should be about unity. We checked that the fits were consistent with the faint magnitude limits of the filters in which the object was not detected. If not, the fit was rejected. The fit with the minimum value of $\chi_{v}^{2}$ was adopted as the best fit and the corresponding values of $E(B-V)$, age and $M_{i}$ were adopted. This method was applied for fits with the four sets of the Starburst99 models and with the Frascati models. Figure 8 shows some examples of the results of the fitting process.

To estimate the uncertainty in the determined parameters we use confidence limits. If $\chi_{v}^{2}<\chi_{v}^{2}(\mathrm{~min})+1$ then the resulting parameters, i.e. $\log (t), \log \left(M_{i}\right)$ and $E(B-V)$, are within the $68.3 \%$ probability range. So the accepted ranges in age, mass 
and extinction are derived from the fits which have $\chi_{v}^{2}(\min )<$ $\chi_{v}^{2}<\chi_{v}^{2}(\min )+1$. With this method we derived the ages, initial masses ${ }^{2}$ and extinction with their uncertainties of 602 clusters.

Figure 9 shows a histogram of number of clusters as function of $E(B-V)$. We will use this distribution for the two dimensional maximum likelihood fit of clusters detected in three bands only. The redding is small: $90 \%$ of the clusters have a reddening lower than $0.40 ; 67 \%$ of the clusters have a reddening lower than 0.18 and $23 \%$ have no detectable reddening.

\subsection{Two dimensional maximum likelihood method}

For the clusters observed in only three bands, it is not possible to make a three dimensional maximum likelihood fit. To reduce the parameter space we adopted the probability distribution of $E(B-V)$ in the range of $E(B-V)$ between 0.0 and 0.4 , as shown in Fig. 9. For every value of $E(B-V)$ between 0.0 and 0.40 (in steps of 0.02 ) and for every age of the model cluster, we first determine the mass of the cluster by means of Eq. (3). The results of the three dimensional fits have shown this is a good approximation. This results in a maximum likelihood age of the cluster for every value of $E(B-V)$.

The $\chi_{v}^{2}$ which comes out from the two dimensional maximum likelihood method is used to distinguish between the accepted and rejected fits. For the parameter $v$ we used a value of $3-1=2$, because we only fit the age of the cluster. The mass of the cluster comes from the scaling of the magnitudes to the best-fit model. A fit is accepted if it agrees with the lower magnitude limits in the filters where it was not measured. To determine the age of the cluster, we average the ages, weighted by the probability that each particular value of $E(B-V)$ occurs, derived by normalizing the distribution in Fig. 9 in the range of $0.0<E(B-V)<0.40$. The error in the age is determined by calculating the value of the standard deviation $\sigma$ of the age, again weighted with the probability that the value of $E(B-V)$ occurs. The value of $E(B-V)$ is determined by using the one which belongs to the model with the age closest to the average age. The initial mass of the cluster is then calculated by Eq. (3) for the adopted value of $E(B-V)$. With this method we derived the age, initial mass and the extinction of 275 clusters, using both the Starburst 99 models and the Frascati models.

\subsection{Starburst99 or Frascati models?}

We have compared the energy distributions of the objects with five sets of models: four sets of Starburst 99 models, with $Z=Z_{\odot}$ or $2 Z_{\odot}$ and $M_{\text {up }}=30$ or $100 M_{\odot}$, and the Frascati models with $Z=Z_{\odot}$ and $M_{\text {up }}=25 M_{\odot}$. Based on the lack of [OIII] emission we adopt the models with $M_{\text {up }}=25$ or $30 M_{\odot}$.

\footnotetext{
2 The initial cluster mass that we derived in this way is in fact "the initial mass of the current stellar population", i.e. it is the "current mass corrected for stellar evolution effects". This value can be different from the the initial mass of the original cluster if the cluster suffered evaporation or disruption. In that case the derived initial mass is the initial mass of the original cluster minus the fraction that has disappeared by evaporation or disruption.
}

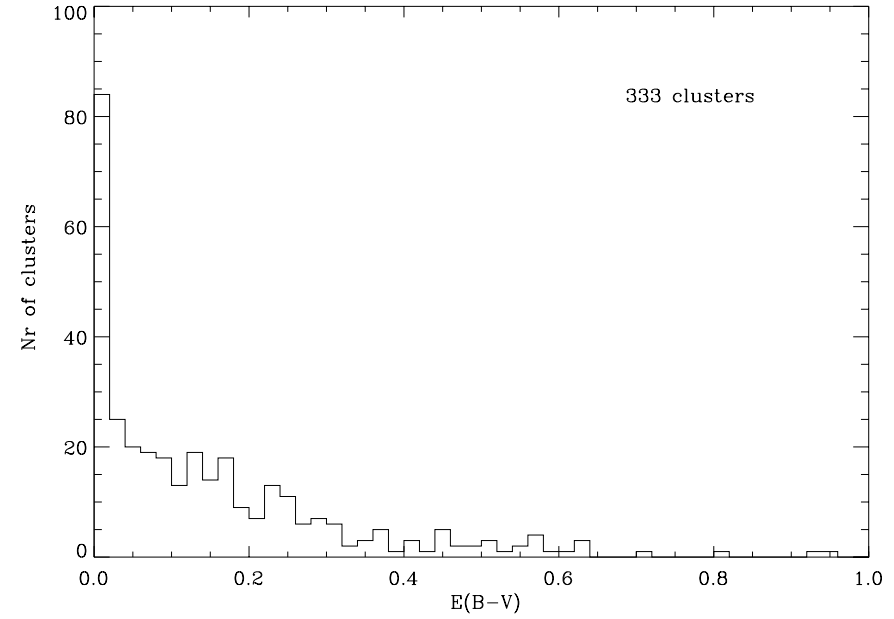

Fig. 9. Histogram of the values of $E(B-V)$ from the fits with observations in $B V R I$ and $U B V R I$. The extinction is small: $90 \%$ of the clusters have $E(B-V)<0.40$.

The models with $M_{\text {up }}=100 M_{\odot}$ are only used later to check the influence of this adopted upper limit on our results.

We have compared the ages derived from fitting the observed SEDs with the Starburst 99 models and the Frascati models, for objects measured in at least four filters and with fits of $\chi_{v}^{2}<3.0$. We found that in the age range of 10 to $800 \mathrm{Myr}$ there is a reasonable correlation between the results of the SB99 models and the Frascati models, with the Frascati models giving ages systematically about 0.4 dex smaller than the SB99 models. In this age range we adopt the SB99 models, because they are based on more reliable evolutionary tracks, better stellar atmosphere models and because the nebular continuum is included. We find that in this mass range the fits with the SB99 models have smaller $\chi_{v}^{2}$ than the fits with the Frascati-models. For objects with ages above about $700 \operatorname{Myrs}(\log (t)>8.85)$ the Frascati-models give the most reliable fits with smaller $\chi_{v}^{2}$ than the SB99 models. This is probably because the lower mass limit of the SB99 models is $1 M_{\odot}$, and the Frascati models go down to $0.6 M_{\odot}$. Moreover the SB99 models do not go beyond 1 Gyr. Based on this comparison we adopt the SB99 fits for ages less than 700 Myrs and the results of the Frascati models for older ages.

\subsection{Uncertainties in the derived parameters}

The determination of the cluster parameters, age, mass and extinction, by means of the two or thee dimensional maximum likelihood fitting method, i.e. the 3(2)DEF-method, results not only in the values of $\log \left(M_{\mathrm{cl}}\right), \log (t)$ and $E(B-V)$ but also in their maximum and minimum acceptable values. The bestfit values are not necessarily in the middle of the minimum and the maximum values. If we define the uncertainties in the parameters, $\Delta E(B-V), \Delta \log \left(M_{\mathrm{cl}}\right)$ and $\Delta \log (t)$ as half the difference between the maximum and mimimum values, we find that 30 percent of the clusters have $\Delta E(B-V)<0.08$, 50 percent have $\Delta E(B-V)<0.11$ and 70 percent have $\Delta E(B-V)<0.15$. For the uncertainties in the mass determination we find $\Delta \log \left(M_{\mathrm{cl}}\right)<0.20,<0.33$ and $<0.55$ for 30,50 
and 70 percent of the clusters respectively. For the uncertainties in the age determination we find $\Delta \log (t)<0.23,<0.39$ and $<0.69$ for 30,50 and 70 percent of the clusters respectively. Taking the values for 50 percent of the clusters as representative, we conclude that the uncertainties are $\Delta E(B-V) \simeq 0.11$, $\Delta \log \left(M_{\mathrm{cl}}\right) \simeq 0.33$ and $\Delta \log (t) \simeq 0.39$.

\subsection{Contamination of the cluster sample by stars?}

To estimate the number of stars that may contaminate our cluster sample we use the stellar population in the solar neighbourhood. From the tabulated stellar densities as a function of spectral type (Allen 1976) we derived the number of stars brighter than a certain value of $M_{V}$ per $\mathrm{pc}^{3}$. Using the mass density of $0.13 M_{\odot} \mathrm{pc}^{-3}$, we derived the number of stars per unit stellar mass. The results are listed in Table 2. The total stellar mass of the observed region of M 51 (see Fig. 2) is estimated to be about $1 / 20$ of the total mass of $5 \times 10^{10} M_{\odot}$ in the disk of that galaxy (Athanassoula et al. 1987), i.e. about $2.5 \times 10^{9} M_{\odot}$. This implies that we can expect the following numbers of stars brighter than $M_{V}<-6.5$ per spectral type in the observed region: $4(\mathrm{O}), 13$ (B), 6 (A), 6 (F), 6 (G), <1 (K) and $<1(\mathrm{M})$. So we can expect of the order of 40 bright stars with $M_{V}<-6.5$ in the observed region of M 51. However, these are all massive young supergiants of which the vast majority will be in clusters! So the number of bright stars outside clusters, that may contaminate our sample of clusters will be considerable smaller, and we expect it to be smaller than about 20 out of the total sample of 877. Moreover, we have shown above, from the lack of O[III] emission, that the clusters in M 51 have a shortage of massive stars with $M>30 M_{\odot}$. We can expect this effect also to occur for the massive field stars. This would reduce the number of expected contaminating stars even further. Tests have shown that a considerable fraction of possibly remaining stellar sources will be eliminated by the requirement that their energy distribution should fit that of cluster models within a given accuracy. Based on all these considerations, we conclude that contamination of our cluster sample with very massive stars of $M_{V}<-6.5$ outside clusters is expected to be negligible.

\section{The mass versus age distribution}

We discuss the results from the fitting of the observed energy distributions to those of cluster models with solar metallicity and with and upper mass limit of $30 M_{\odot}$. We have checked that the fits with twice solar metallicity and with an upper limit of $100 M_{\odot}$ give about the same results.

Figure 10 shows the mass versus age distribution of sources with $M_{v}<-7.5$ (to eliminate possible stellar sources) and with energy distributions that could be fitted to that of a cluster models with an accuracy of $\chi_{v}^{2} \leq 3.0$. (The distributions for clusters with $\chi_{v}^{2} \leq 1$ or 10 , not shown here, show the same distribution, but with 294 and 508 clusters respectively.) We see that the lower limit of the mass increases with increasing age, from about $1000 M_{\odot}$ at $t \simeq 5$ Myrs to about $5 \times 10^{4} M_{\odot}$ at $1 \mathrm{Gyr}$. This is due to the expected effect of fading of the clusters as they age (see Sect. 4.1). The full line in the figure is the fading line in the $R$ magnitude for clusters which have a limiting

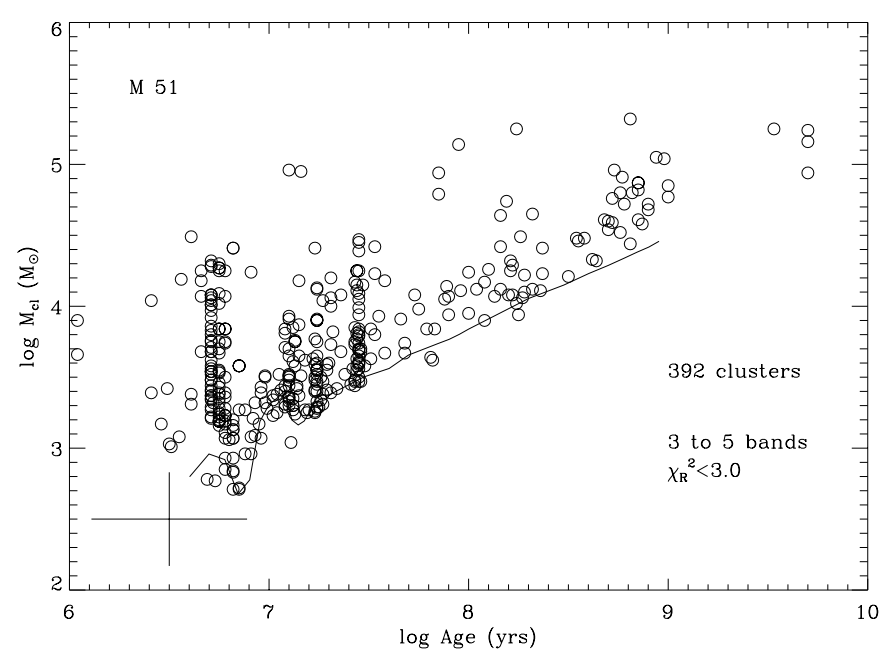

Fig. 10. The mass versus age relation of the 392 clusters with $M_{V}<$ -7.5 , whose energy distribution could be fitted with an accuracy of $\chi_{v}^{2}<3.0$. The cross shows the characteristic uncertainties (Sect. 5.5). The full line is the predicted fading line due to the evolution of clusters with $E(B-V)=0$ and with a limiting magnitude of $R_{\lim }=22.0$ at the distance of M 51. This line roughly agrees with the observed lower limit. The distribution is discussed in the text.

magnitude of $R_{\lim }=22.0$ at the distance of M 51 for a reddening of $E(B-V)=0$. The line in Fig. 10 thus gives the mass of the clusters, that reaches this magnitude detection limit, as a function of age.

These initial mass versus age distribution of the clusters in Fig. 10 show the following characteristics:

- (i) The lower mass limit increases with age due to the fading of the clusters. The observed lower limit agrees with the predicted ones for the $R$ band. There are even hints of the presence of the predicted dips in the lower limit near $\log (t)=6.8$ and 7.2. This strengthens our confidence in the adopted models.

- (ii) There are clear concentrations in the distribution at $\log (t)=6.70$ and 7.45 and possibly also around $\log (t) \simeq 7.2$. These are due to the properties of the cluster models and the adopted method. In Fig. 7 we have shown that the colours of the models do not change monotonically with time, but that there are phases when the colours change rapidly with time. These phases occur in the range of $6.5<\log (t)<7.3$. Large changes in the colours of the models occur just before and after the peaks where the slopes of the curves in Fig. 7 are large. It is more difficult to fit the observed energy distributions with high accuracy to models in the age range when the spectral changes are large, than in the age range when the changes are small. So there is a tendency of the model fits to concentrate in agebins just outside the age-regions of large spectral changes. This explains the concentrations and the voids in the derived age distributions of the clusters between about $6.5<\log (t)<7.5$.

- (iii) The density of the points drops at $\log (t)>7.5$. Since the age scale is logarithmic, we might have expected an increasing density of points towards the higher ages, which is not observed. This is due to the disruption or dispersion of the clusters (see first paragraphs of Sect. 7). 
Table 2. Numbers of stars above a certain absolute magnitude limit in the solar neighbourhood.

\begin{tabular}{ccccccccc}
\hline \hline Limit & $\mathrm{O}$ & $\mathrm{B}$ & $\mathrm{A}$ & $\mathrm{F}$ & $\mathrm{G}$ & $\mathrm{K}$ & $\mathrm{M}$ & Total \\
\hline$M_{V}<-5.5$ & -8.3 & -7.6 & -8.0 & -7.4 & -7.4 & -8.5 & -8.5 & -6.9 \\
$M_{V}<-6.5$ & -8.8 & -8.3 & -8.6 & -8.6 & -8.6 & $<-9.5$ & $<-9.5$ & -7.9 \\
$M_{V}<-7.5$ & -9.8 & -9.3 & -9.6 & -9.6 & -9.6 & $<-10.5$ & $<-10.5$ & -8.9 \\
\hline
\end{tabular}

(1) The data are in $\log \left(\right.$ Number/per $\left.M_{\odot}\right)$.

(2) The number of stars with $M_{V}<-7.5$ was estimated to be about 10 times smaller than for $M_{V}<-6.5$ because of the small number of very massive stars formed, and their short evolution time.

We conclude that the mass versus age distribution agrees with the expected evolutionary fading of the clusters and that the decrease in numbers of clusters with age shows the affect of disruption/dispersion of clusters with time. The concentrations of the clusters at ages around $\log (t)=6.8,7.2$ and 7.45 are due to statistical effects and do not represent periods of enhanced cluster formation.

\section{The cluster initial mass function}

The data in Fig. 10 and the detailed study of this distribution by Boutloukos \& Lamers (2002) show that the clusters in the inner spiral arms of M 51 disrupt on a time scale of about tens of Myrs. In fact, these authors derived the dependence of the disruption time on the initial mass of the clusters in the inner spiral arms of M 51 as

$\log t_{\mathrm{disr}}=\log t_{4}+\gamma \times \log \left(M_{\mathrm{cl}} / 10^{4} M_{\odot}\right)$

with $\log t_{4}=7.64 \pm 0.22$ and $\gamma=0.62 \pm 0.06$ for the mass range of $3 \leq \log \left(M_{\mathrm{cl}} / M_{\odot}\right) \leq 5.2$, where $M_{\mathrm{cl}}$ is the initial mass of the cluster. We see that clusters with an initial mass larger than $10^{4} M_{\odot}$ survive $4 \times 10^{7}$ years. Clusters with an initial mass of only $10^{3} M_{\odot}$ disrupt on a time scale of $1 \times 10^{7} \mathrm{yrs}$. This implies that the cluster initial mass function cannot be derived from the total sample of clusters, because the disruption will produce a strong bias towards the more massive clusters. However for the youngest clusters with ages less than about $10 \mathrm{Myr}$ disruption is not yet an important effect and, therefore, these clusters can be used to derive the initial cluster mass function.

Figure 11 shows the resulting mass distribution of clusters with an age less or equal to $10 \mathrm{Myr}$ for two samples of clusters. The first sample contains all 354 clusters younger than $10 \mathrm{Myr}$. The second sample contains 168 clusters in the same age range but with a mass determination of $\Delta \log \left(M_{\mathrm{cl}}\right) \leq 0.25$. Both samples show the same characteristics: a steep increase in number between $2.5<\log M_{\mathrm{cl}}<3.0$ and a slow decrease to higher masses. The steep increase is due to the detection limit or the disruption of the low mass clusters. The slow decrease reflects the cluster initial mass function (CIMF). The decrease indicates that the clusters are formed with an CIMF that has a negative slope of $\mathrm{d} \log (N) / \mathrm{d} \log (M)$, as expected.

If the CIMF can be written as a power law of the type

$N(M) \mathrm{d} M \sim M^{\alpha} \mathrm{d} M \quad$ for $M_{\min }<M<M_{\max }$

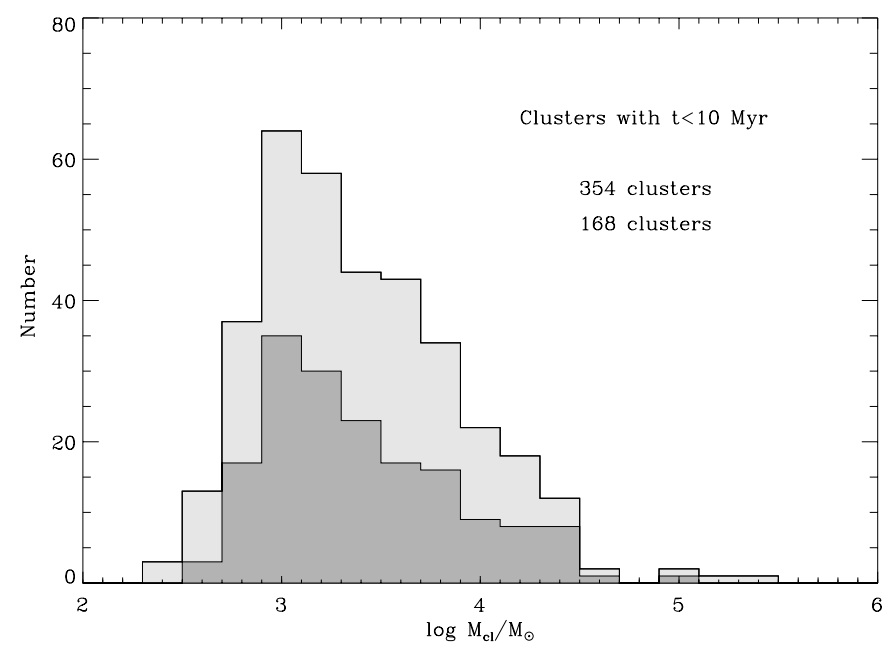

Fig. 11. Histograms of the mass of clusters (in $\log M_{\mathrm{cl}}$ ) with an age of 10 Myrs or younger. The light shaded area shows the distribution of all 354 clusters. The dark shaded area shows the histogram of clusters with an accurate mass determination of $\Delta \log M_{\mathrm{cl}}<0.25$. The steep part at $\log (M)<3.0$ is due to the detection limit. The decrease at $\log (M)>3.0$ reflects the slope of the cluster IMF. (All masses have to be increased by a factor 2.1 if the lower limit of the stellar mass is $0.2 M_{\odot}$ rather than the value of $1 M_{\odot}$ that was adopted in the Starburst99 models.)

then the normalized cumulative distribution will be

$\Sigma(M)=A-B \times M^{-\alpha+1} \quad$ with

$A=B \times M_{\text {min }}{ }^{-\alpha+1}$

$B=\left(M_{\min }^{-\alpha+1}-M_{\max }^{-\alpha+1}\right)^{-1}$.

Figure 12 shows the observed and predicted normalized cumulative distribution of the 149 clusters with an age less than $10 \mathrm{Myr}$ and with a mass $M_{\mathrm{cl}}>2.5 \times 10^{3} M_{\odot}$ that are used for the determination of the CIMF. We eliminated the clusters with $M_{\mathrm{cl}}<2.5 \times 10^{3} M_{\odot}$ from the sample because Fig. 11 shows that the sample may not be complete for smaller masses. The cumulative distributions of the 84 clusters of $M>2.5 \times 10^{3} M_{\odot}$, younger than $10 \mathrm{Myr}$, which are fitted to the models with an accuracy of $\chi_{v}^{2} \leq 3.0$, or of the 66 clusters with $\Delta \log \left(M_{\mathrm{cl}}\right) \leq 0.25$, not shown here, (where $\Delta \log \left(M_{\mathrm{cl}}\right)$ is the uncertainty in the mass determination), have the same shape as the distribution in Fig. 12. For these three samples we have determined the values of $\alpha$ and $M_{\min }$ by means of a linear regression under the reasonable assumption that $M_{\min } \ll M_{\max }$, which implies that 
Table 3. The CIMF for clusters with $t<10 \mathrm{Myr}$ and $M_{\mathrm{cl}}<2.5 \times$ $10^{3} M_{\odot}$.

\begin{tabular}{lrcc}
\hline \hline Sample & $\mathrm{Nr}$ & $\log M_{\min }$ & $\alpha$ \\
\hline All & 149 & 3.49 & $2.12 \pm 0.26$ \\
$\Delta \log M_{\mathrm{cl}}<0.25$ & 66 & 3.48 & $2.04 \pm 0.41$ \\
$\chi_{v}^{2}<3.0$ & 82 & 3.46 & $2.16 \pm 0.40$ \\
\hline
\end{tabular}

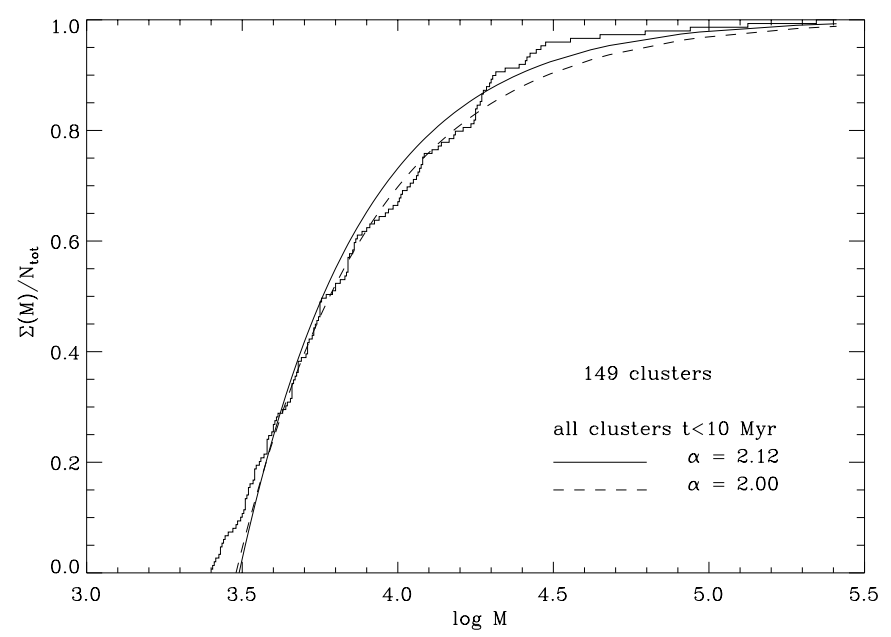

Fig. 12. The cumulative mass distribution of 149 clusters with an age less than 10 Myrs and an inital mass of $\log (M)>3.40$. The full line is the best power-law fits for an IMF with the value of $\alpha$ and $M_{\min }$ from Table 3. The dashed line is the fit with $\alpha=2.00$.

$A \simeq 1$. The resulting values of $\alpha$ and $M_{\min }$ are listed in Table 3 . The table shows that $\alpha \simeq 2.1$ for all three samples.

The predicted cumulative distribution with the derived values of $\alpha=2.12$ and $M_{\min }$ is shown in Fig. 12 (full line). The figure shows a slight underabundance of clusters in the range of $4.0<\log \left(M_{\mathrm{cl}}\right)<4.3$ and a slight overabundance in the range of $\log \left(M_{\mathrm{cl}}\right)>4.3$. In fact, for the mass range of $3.5<$ $\log \left(M_{\mathrm{cl}}\right)<4.3$ a fit with $\alpha=2.00$, shown by dashed lines, fits the distribution excellently. We conclude that the CIMF of clusters younger than $10 \mathrm{Myr}$ has a slope of $\alpha \simeq 2.1 \pm 0.3$ in the mass range of $3.0<\log \left(M_{\mathrm{cl}}\right)<5.0$ and a slope of $2.00 \pm 0.05$ in the range of $3.0<\log \left(M_{\mathrm{cl}}\right)<4.3 M_{\odot}$.

The derived exponent of the cluster IMF is very similar to the value of $\alpha=2.0$ of young clusters in the Antennae galaxies, as found by Zhang \& Fall (1999). It shows that the IMF of clusters formed in the process of galaxy-galaxy interaction is very similar to the one of clusters formed in the spiral arms of a galaxy, long after the interaction. This mass distribution is also similar to that of giant molecular clouds (e.g. McKee 1999; Myers 1999). This may support the suggestion that the mass distribution of the clusters is determined by the mass distribution of the clouds from which they originate.

\section{The cluster formation history}

One of the goals of this paper is to study the influence of the interaction between M 51 and its companion on the cluster formation in the region of the inner spiral arms. To this purpose we compare the observed age distribution of the clusters with

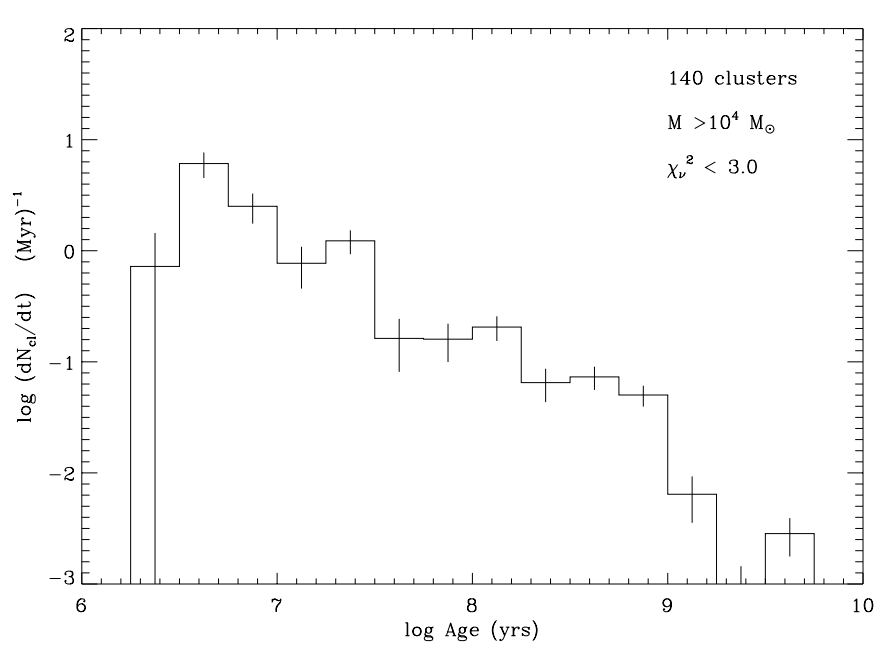

Fig. 13. The formation rates of the observed clusters with an initial mass of $M_{\mathrm{i}}>10^{4} M_{\odot}$ in number per Myr, for clusters with an accuracy of the fit of the energy distribution of $\chi_{v}^{2} \leq 3.0$. The $1 \sigma$ uncertainty due to the Poisson statistics in each bin $(\sigma=1 / \sqrt{(} N))$ is indicated. The diagram shows that the formation rate of the observed clusters decreases with age. This is due to the disruption of the older clusters.

predictions for a constant cluster formation rate. This comparison is hampered by two effects: (a) the disruption of clusters and (b) the fading of clusters below the detection limit. The disruption time of the clusters in the inner spiral arms of M 51 is given by Eq. (4). We see that only clusters with an initial mass larger than about $10^{4} M_{\odot}$ will survive more than 40 Myrs.

Figure 13 shows the histogram of the formation rates of the detected clusters, in number per Myr, for 140 clusters with $M_{\mathrm{cl}}>10^{4} M_{\odot}$ and with an energy distribution that is fitted with an accuracy of $\chi_{v}^{2} \leq 3.0$. The sample of 111 clusters with $M_{\mathrm{cl}}>10^{4} M_{\odot}$ and with an age uncertainty of $\Delta \log (t)<0.25$, and the full sample of 285 clusters with $M_{\mathrm{cl}}>10^{4} M_{\odot}$, not shown here, have a distribution very similar to the one shown in Fig. 12.

All three samples show about the same characteristics.

(a) There is a general trend of a decrease in the cluster formation rate towards increasing age.

(b) There is a steep drop around $\log (t) \simeq 7.5$. This drop is the result of the concentration of clusters at ages $\log (t)=7.45$ that was apparent in the mass versus age diagrams of Fig. 10, and was explained in Sect. 6.

(c) There is no clear evidence for a peak in the cluster formation rate near $\log (t) \simeq 8.6$, which is the time of the interaction of M51 with its companion, and which is the age of the huge starburst in the nucleus of M 51. There is a hint of a small peak around $\log (t) \simeq 8.1$. However this peak is only $2 \sigma$ high. Its reality has to be verified with a larger sample of clusters.

(d) The peak in the last bin of 5 Gyr contains all the clusters ages older than $3 \mathrm{Gyr}$, because the cluster models that we used for fitting the energy distributions do not go beyond $5 \mathrm{Gyr}$.

We have checked that these characteristics are not the result of the binning process: the same features appear for different choices of the binning parameters. 
The general decrease in the formation rate of the observed clusters is partly due to the evolutionary fading of the clusters and partly due to the disruption of clusters. In Sect. 6 and in Fig. 10 we have shown that clusters with an initial mass of $10^{4} M_{\odot}$ fade below the detection limit when they are older than $\log (t)=8.2$. This can explain the decrease in the formation rate of clusters older than about 150 Myrs. However, clusters with $M_{\mathrm{cl}}>10^{4} M_{\odot}$ and younger than about $100 \mathrm{Myr}$ should still all be detectable. The fact that we see a decrease in the apparent cluster formation rate must thus be due to the disruption of clusters (unless for some unknown reason the cluster formation rate has been steadily increasing from 100 Myrs up to now, which we consider unlikely).

It is interesting that the CFR in the inner spiral arms of $\mathrm{M} 51$, at a distance of about 1 to $3 \mathrm{kpc}$ from the nucleus, does not show any evidence for a peak at the age of the strongest interaction of M 51 with its companion galaxy. The closest approach occurred about 250-400 Myrs ago according to the dynamical models of Barnes (1998) or 400-500 and 50-100 Myrs ago according to Salo \& Laurikainen (2000). The only significant peak occurred at $\log (t) \simeq 7$.4. However we attributed this peak to the large changes in the energy distributions of the models in the age range of $6.5<\log (t)<7.5$, and not to a real increase in the CFR.

\section{Summary and conclusions}

We have studied images of the inner spiral arms of the interacting galaxy M 51 obtained with the HST-WFPC2 camera in broad-band $U B V R I$ and in the narrow band $\mathrm{H} \alpha$ and [OIII] filters. The study can be summarized as follows:

1. We found a total of 877 point-like objects, which are probably clusters. Many of the clusters are strong $\mathrm{H} \alpha$ emitters, but none of the clusters, not even the youngest ones, have an excess of radiation in the [OIII] line at $5007 \AA$ (F502Nfilter). This suggests that the upper mass limit of the stars in the clusters is about 25 to $30 M_{\odot}$.

2. We have compared their energy distributions with those of Starburst99 cluster models (Leitherer et al. 1999) for instantaneous star formation with a stellar IMF of exponent 2.35, solar metallicity, a lower and upper stellar mass limit of $1 M_{\odot}$ and $30 M_{\odot}$ respectively. The energy distributions were also compared with those of the Frascati models (Romaniello 1998). For clusters younger than $700 \mathrm{Myr}$ the results from the fitting with the Starburst99 models were adopted because these models are more accurate for young clusters and the fits of the energy distributions are better than those of the Frascati models. For older clusters the results from the fits with the Frascati models were adopted.

3. For clusters that were observed in four or five bands a three dimensional maximum likelihood method was used to derive the properties of the clusters from the comparison between the observed and predicted energy distributions. The free parameters are the age $t$ and $E(B-V)$, which together determined the shape of the energy distribution, and the initial cluster mass $M_{\mathrm{cl}}$ which determines the absolute magnitude. For clusters that were not observed in all bands, the empirically derived lower magnitudes limits were taken into account.

4. For clusters that were observed in only three bands the age and mass were derived in a two-dimensional maximum likelihood fitting of the energy distributions, with $t$ and $M_{\mathrm{cl}}$ as free parameters. The observed probability distribution of $E(B-V)$ was used as a weighting factor in the fitting procedure.

5. The histogram of $E(B-V)$ is strongly peaked at very small $E(B-V) \simeq 0$. All cluster have a reddening smaller than $E(B-V)<1.0$ and $67 \%$ of the clusters have $E(B-V)<0.18$.

6. We have analysed the observed clusters also with cluster models of higher metallicity, $Z=2 \times Z_{\odot}$. These higher metallicity models fit the observations considerably worse than the solar metallicity models. For instance, for solar metallicity models the energy distribution of 294 clusters can be fitted with an accuracy of $\chi_{v}^{2} \leq 1.0$ and 392 with $\chi_{v}^{2} \leq 3.0$. For models with twice the solar metallicity these numbers are respectively 138 and 217 . So the energy distributions of the clusters support the adopted solar metallicity.

7. The clusters have masses in the range of $2.5<\log \left(M_{\mathrm{cl}}\right)<$ 5.7 and ages of $\log (t)>5.0$. These masses are the initial masses of the clusters, i.e. the current mass corrected for stellar evolution effects, but not corrected for evaporation or disruption. All derived masses have to be multiplied by a factor 1.3 if the lower mass of the stars is $0.6 M_{\odot}$, instead of the adopted $1 M_{\odot}$, and by a factor 2.1 if the lower mass is $0.2 M_{\odot}$, as found for the Orion Nebula cluster.

8. The distribution of the clusters in a mass-versus-age diagram shows the predicted lower limit due to the evolutionary fading of the clusters, including the dips at $\log (t) \simeq 6.8$ and 7.1. Three apparent concentrations at $\log (t)=6.7,7.2$ and 7.45 are not real but due to the properties of the cluster models used.

9. About $60 \%$ of the clusters are younger than 40 Myr. The number of older clusters is much less than expected for a constant cluster formation rate. This is partly due to the evolutionary fading of low mass clusters below the detection limit, and partly due to the disruption of the clusters.

10. The cluster initial mass function (CIMF) was derived from the cumulative mass distribution of clusters younger than $10 \mathrm{Myr}$, for which disruption has not occured. The CIMF has a slope of $\alpha=2.1 \pm 0.3$ in the range of $3.0<\log \left(M_{\mathrm{cl}}\right)<5.0$ and $\alpha=2.00 \pm 0.05$ in the range of $3.0<\log \left(M_{\mathrm{cl}}\right)<4.5 M_{\odot}$, for $N\left(M_{\mathrm{cl}}\right) \sim M_{\mathrm{cl}}{ }^{-\alpha}$. This slope is the same to that found in the interacting Antennae galaxies (Zhang \& Fall 1999). Zhang and Fall deived a power law slope of the CIMF of $\alpha=1.95 \pm 0.03$ and $2.00 \pm 0.04$ for two cluster samples of the Antennae galaxies. The good agreement between these slopes and the one found by us suggests that $\alpha$ is about the same for cluster formation triggered by strong galaxy-galaxy interactions, such as presently going on in the Antennae, as for cluster formation that is not dominated by the interactions.

11. The age distribution of clusters with $M_{\mathrm{cl}}>10^{4} M_{\odot}$, is used to derive the history of the cluster formation rate (CFR). There is a general trend of a decrease of the formation rate 
of the observed clusters with age. It is unlikely that the real CFR has been increasing continuously from about $1 \mathrm{Gyr}$ to the present time. The decrease of the CFR with age of clusters younger than about $100 \mathrm{Myr}$ cannot be due to evolutionary fading, but it is due to the disruption of clusters. For clusters older than $200 \mathrm{Myr}$ the decrease of the derived CFR could, at least partly, be due to evolutionary fading.

12. There is no evidence for a peak in the CFR at about $400 \mathrm{Myr}$, which is the time of the interaction of M 51 with its companion and the age of the huge starburst in the nucleus.

In a forthcoming paper we describe the cluster formation as a function of location in a large part of M 51, using the same methods as used here (Bastian et al. 2002). The disruption of clusters in M 51, derived from the results of the study presented here, are described by Boutloukos \& Lamers (2002).

Acknowledgements. H.J.G.L.M.L. and N.B. are grateful to the Space Telescope Scence Institute for hospitality and financial support during several stays. We thank Claus Leitherer for help and advice in the calculation of the cluster models. Support for the SINS program GO-9114 was provided by NASA through a grant from the Space Telescope Science Institute, which is operated by the Association of Universities for Research in Astronomy, Inc. under NASA contract NAS 5-26555. N.B. ackowledges a grant from the Netherlands Organization for Scientific Research. We thank the unknown referee for constructive comments that resulted in an improvement of this paper.

\section{References}

Allen, C. W. 1976, Astrophysical Quantities (London: the Athlone Press), 247

Athanassoula, E., Bosma, A., \& Papaioannou, P. 1987, A\&A, 179, 23

Barnes, J. E. 1998, in Galaxies: Interactions and Induced Star Formation, ed. R. C. Kennicutt, F. Schweizer, \& J. E. Barnes, Saas Fee Advanced Course 26 (Berlin: Springer Verlag), 275

Bastian, N. R., \& Lamers, H. J. G. L. M. 2002, in preparation Boutloukos, S. G., \& Lamers, H. J. G. L. M. 2002, MNRAS, accepted Brocato, E., \& Castellani, V. 1993, ApJ, 410, 99

Cassisi, S., Castellani, V., \& Straniero, O. 1994, A\&A, 282, 753
Chiosi, C., \& Maeder, A. 1986, ARA\&A, 24, 329

Diaz, A. I., Terlevich, E., Vilchez, J. M., Pagel, B. E. J., \& Edmunds, M. G. 1991, MNRAS, 253, 245

Feldmeier, J. J., Ciardullo, R., \& Jacoby, G. H. 1997, ApJ, 479, 231

Hernquist, L. 1990, in Dynamics and Interactions of Galaxies, ed. R. Wielen (Berlin: Springer Verlag), 108

Hill, J. K., Waller, W. H., Cornett, R. H., et al. 1997, ApJ, 477, 673

Hillenbrand, L. A. 1997, AJ, 113, 1733

Holtzman, J. A., Burrows, C. J., Casertano, S., et al. 1995, PASP, 107, 156

Kennicutt, R. C. 1998, in Galaxies: Interactions and Induced Star Formation, ed. R. C. Kennicutt, F. Schweizer, \& J. E. Barnes, Saas Fee Advanced Course 26 (Berlin: Springer Verlag), 1

Kurucz, R. L. 1993, Kurucz CD-ROM 13, ATLAS9 Stellar Atmosphere Programs and the $2 \mathrm{~km} \mathrm{~s}^{-1}$ grid (Cambridge: SAO)

Lamers, H. J. G. L. M., Panagia, N., Scuderi, S., et al. 2002, ApJ, 566, 818

Leitherer, C., Schaerer, D., Goldader, J. D., et al. 1999, ApJS, 123, 3

Lejeune, T., Buser, R., \& Cuisinier, F. 1997, A\&AS, 99, 173

McKee, C. F. 1999, in The origin of stars and planetary systems, ed. C. J. Lada, \& N. D. Kylafis (Dordrecht: Kluwer Acad. Publ.), 29

Millard, J., Branch, D., Hatano, K., et al. 1999, ApJ, 527, 746

Myers, P. C. 1999, in The origin of stars and planetary systems, ed. C. J. Lada, \& N. D. Kylafis (Dordrecht: Kluwer Acad. Publ.), 67

Panagia, N. 2000, in The Chemical Evolution of the Milky Way, ed. F. Matteucci, \& F. Giovannelli (Dordrecht: Kluwer Acad. Publ.), 495

Romaniello, M. 1998, Ph.D. Thesis: Young stellar Populations in the Large Magelanic Cloud; surprises from HST observations, Scuola Normale Superiore di Pisa

Salo, H., \& Laurikainen, E. 2000, MNRAS, 319, 377

Schmutz, W., Leitherer, C., \& Gruenwald, R. 1992, PASP, 104, 1164

Schweizer, F. 1998, in Galaxies: Interactions and Induced Star Formation, ed. R. C. Kennicutt, F. Schweizer, \& J. E. Barnes, Saas Fee Advanced Course 26 (Berlin: Springer Verlag), 105

Scuderi, S., Panagia, N., Capetti, A., et al. 2002, ApJ, in press

Stetson, P. B. 1987, PASP, 99, 191

Toomre, A., \& Toomre, J. 1972, ApJ, 178, 623

Voit, M. 1997, HST Data Handbook, Space Telescope Science Institute

Whitmore, B. C., \& Heyer, I. 1997, WFPC2 ISR 97-08, Space Telescope Science Institute

Whitmore, B. C., Zhang, Q., Leitherer, C., et al. 1999, AJ, 118, 1551

Zhang, Q., \& Fall, S. M. 1999, ApJ, 527, L81 